\title{
A MINIMAL DECAY RATE FOR SOLUTIONS OF STABLE $n$ TH ORDER HOMOGENEOUS DIFFERENTIAL EQUATIONS WITH CONSTANT COEFFICIENTS
}

\author{
DAVID W. KAMMLER
}

ABSTRACT. In this paper we establish the existence of an envelope function (depending only on $n$ and $\alpha>0$ ) which provides a pointwise bound on the size of any normalized solution $y$ of any homogeneous $n$th order differential equation with constant coefficients for which the roots of the corresponding characteristic polynomial have real parts which do not exceed $-a$. An explicit representation for this envelope is obtained in the special case where these roots are further constrained to be real valued.

1. Introduction. For $n=1,2, \ldots$ and for $\alpha>0$ we define $L_{n \alpha}$ to be the set of all possible complex valued solutions of all possible $n$th order homogeneous differential equations

$$
\left[\left(D-\lambda_{1}\right)\left(D-\lambda_{2}\right) \cdots\left(D-\lambda_{n}\right)\right] y(t)=0, \quad t \geq 0, D=d / d t
$$

for which

$$
\operatorname{Re} \lambda_{i} \leq-\alpha, \quad i=1,2, \cdots, n
$$

When $y \in L_{n a^{\prime}} y(t)$ decays to zero as $t \rightarrow+\infty$ so that

$$
\|y\|=\max \{|y(t)|: t \geq 0\}
$$

provides a well-defined measure of the overall size of $y$.

In this paper we show that the parameters $n, \alpha$, and $\|y\|$ are sufficient to characterize a minimal decay rate for such a function $y$.

2. The existence of the envelope function. This minimal decay rate can be bounded by means of an envelope function in the following sense.

Theorem 1. For each $n=1,2, \cdots$ there exists a nonincreasing func. tion $g_{n}:[0,+\infty) \rightarrow(0,1]$ with

Received by the editors June 22, 1973 and, in revised form, March 7, 1974 . AMS (MOS) subject classifications (1970). Primary 34C10, 41A50.

Key words and phrases. Minimal decay rate for exponential sums, Chebyshev polynomial on $[0,+\infty)$ with exponential weight.

Copyright $\odot 1975$, American Mathematical Society 


$$
g_{n}(t) \downarrow 0 \text { as } t \rightarrow+\infty
$$

such that the inequality

$$
|y(t)| \leq\|y\| \cdot g_{n}(\alpha t), \quad t \geq 0,
$$

holds for every $y \in L_{n a}$.

Proof. We define $g_{n}:[0,+\infty) \rightarrow(0,1]$ by

$$
g_{n}(t)=\sup \left\{|y(t)|: y \in L_{n 1},\|y\|=1\right\}, \quad t \geq 0,
$$

and show that $g_{n}$ satisfies the requirements of the theorem. Using (6) and the definition of $L_{n a}$ we see that

$$
\begin{aligned}
g_{n}(\alpha t) & =\sup \left\{|y(\alpha t)|: y \in L_{n 1},\|y\|=1\right\} \\
& =\sup \left\{|y(t)|: y \in L_{n a},\|y\|=1\right\}
\end{aligned}
$$

so that (5) holds whenever $y \in L_{n a}$. Using (7) we see that $g_{n}\left(\alpha^{\prime} t\right) \leq g_{n}(t)$ whenever $L_{n \alpha^{\prime}} \subseteq L_{n 1}$, and since this inclusion holds for all $\alpha^{\prime} \geq 1$ we conclude that $g_{n}$ is nonincreasing.

To complete the proof we must establish (4). From (6) we infer the existence of a sequence $\left\{y_{m}\right\}$ from $L_{n 1}$ such that

$$
g_{n}(m) \leq 2 y_{m}(m), \quad\left\|y_{m}\right\|=1, \quad m=1,2, \cdots .
$$

Defining

$$
u_{m}(t)=y_{m}(m t), \quad t \geq 0, m=1,2, \cdots,
$$

we see that

$$
u_{m} \in L_{n m}, \quad\left\|u_{m}\right\|=1, \quad m=1,2, \cdots .
$$

In [1, Lemma 2] it is shown that a sequence $\left\{u_{m}\right\}$ with the properties (10) converges uniformly to zero on compact subsets of $(0,+\infty)$ and using this together with (8)-(9) we see that as $m \rightarrow \infty$

$$
\varlimsup \lim g_{n}(m) \leq 2 \varlimsup \lim y_{m}(m)=2 \varlimsup \overline{\lim } u_{m}(1)=0
$$

from which (4) follows since $g_{n}$ is nonincreasing.

Since the function $y(t)=\exp (-t)$ is contained in $L_{n 1}$ we have $g_{n}(0)$ $=1$ and by using (4) we see that

$$
T_{n}=\sup \left\{t \geq 0: g_{n}(t)=1\right\}
$$

is finite for each $n=1,2, \ldots$. This being the case each $y \in L_{n a}$ takes 
its maximum modulus somewhere in a finite interval which depends only on $n$ and $\alpha$.

Corollary 1. Let $y \in L_{n \alpha}$ with $\|y\|>0$. Then

$$
\|y\|=\max \left\{|y(t)|: 0 \leq t \leq T_{n} / \alpha\right\}
$$

with

$$
|y(t)|<\|y\|
$$

for all $t>T_{n} / \alpha$.

Proof. By using (11) and (5) we obtain (12) and (13).

Corollary 2. Let $A$ be an $n \times n$ matrix of complex numbers with eigenvalues $\lambda_{1}, \lambda_{2}, \cdots, \lambda_{n}$ which satisfy (2), let $\mathrm{y}:[0,+\infty) \rightarrow \mathrm{C}^{n}$ satisfy the differential equation

$$
D \mathbf{y}(t)=A \mathbf{y}(t), \quad t \geq 0, D=d / d t,
$$

and let $\|\mathbf{y}\|=\max \left\{|\mathbf{y}(t)|_{\infty}: t \geq 0\right\}$ where

$$
|\mathrm{y}(t)|_{\infty}=\max \left\{\left|y_{1}(t)\right|,\left|y_{2}(t)\right|, \cdots,\left|y_{n}(t)\right|\right\}
$$

and where $y_{1}, y_{2}, \cdots, y_{n}$ are the components of $\mathbf{y}$. Then

$$
|\mathbf{y}(t)|_{\infty} \leq\|\mathbf{y}\| g_{n}(\alpha t), \quad t \geq 0
$$

Proof. Using (14) together with the Cayley-Hamilton theorem we find $\left[\left(D-\lambda_{1}\right)\left(D-\lambda_{2}\right) \cdots\left(D-\lambda_{n}\right)\right] \mathrm{y}(t)=\left[\left(A-\lambda_{1} I\right)\left(A-\lambda_{2} I\right) \cdots\left(A-\lambda_{n} I\right)\right] \mathrm{y}(t)=0$ so that each component of $y$ satisfies (1) and (2). This being the case (15) follows at once from (5).

3. The special case of real exponential parameters. We define $\Lambda_{n a}$ to be the set of those real valued functions $y \in L_{n a}$ which satisfy some differential equation (1) in which each $\lambda_{i}$ is real, and we let

$$
\gamma_{n}(t)=\sup \left\{|y(t)|: y \in \Lambda_{n 1},\|y\|=1\right\}, \quad t \geq 0,
$$

denote the corresponding envelope function so that Theorem 1 and its corollaries hold when $L_{n a}, g_{n}$ are replaced by $\Lambda_{n a}, \gamma_{n}$, respectively. We shall present three lemmas which lead to a useful alternative representation for $\gamma_{n}$

Lemma 1. Given $n=1,2, \ldots$ and $\tau \geq 0$ there exists some $y \in \Lambda_{n 1}$ 
with $\|y\|=1$ such that $y(\tau)=\gamma_{n}(\tau)$, i.e., the supremum in (16) is actually a maximum.

Prooof. The exponential function $y(t)=\exp (-t)$ satisfies the lemma when $\tau=0$. In the remainder of the proof we will assume that $\tau>0$. From (16) we infer the existence of a normalized sequence $\left\{y_{m}\right\}$ from $\Lambda_{n 1}$ such that $\left\{y_{m}(\tau)\right\}$ converges to $\gamma_{n}(\tau)$. In [1, Lemmas 1,2$]$ it is shown that from such a normalized sequence from $\Lambda_{n 1}$ we may extract a subsequence which converges uniformly on compact subsets of $(0,+\infty)$ to some $y \in \Lambda_{n 1}$ with $\|y\| \leq 1$. For this choice of $y$ we have

$$
\gamma_{n}(\tau)=\lim y_{m}(\tau)=y(\tau) \leq\|y\| \cdot \gamma_{n}(\tau) \leq \gamma_{n}(\tau)
$$

so that $\|y\|=1$ and $y(\tau)=\gamma_{n}(\tau)$.

As the pointwise supremum of a family of continuous real valued functions, $\gamma_{n}$ is lower semicontinuous. Using Lemma 1 we can show that $\gamma_{n}$ is also upper semicontinuous and thus continuous. (Indeed, let $\left\{t_{m}\right\}$ be any sequence of nonnegative real numbers with limit $t>0$ and for each $m=$ $1,2, \ldots$ let $y_{m} \in \Lambda_{n 1}$ be chosen such that $y_{m}\left(t_{m}\right)=\gamma_{n}\left(t_{m}\right)$ and $\left\|y_{m}\right\|=1$. After passing to a subsequence, if necessary, we may assume that $\left\{y_{m}\right\}$ converges uniformly on compact subsets of $(0,+\infty)$ to some $y \in \Lambda_{n 1}$ with $\|y\| \leq 1$, cf. [1, Lemmas 1, 2], so that

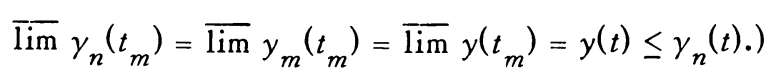

We conjecture that Lemma 1 also holds in the case where $\Lambda_{n 1}, \gamma_{n}$ are replaced by $L_{n 1}, g_{n}$, respectively, and that the (lower semicontinuous) envelope function $g_{n}$ is likewise continuous.

Lemma 2. Let $y \in \Lambda_{n 1}$ with $\|y\|=1$ and assume that

$$
y(\tau)=\gamma_{n}(\tau)<1
$$

for some $\tau>0$. Then $y$ may be written in the form

$$
y(t)=q(t) \cdot \exp (-t), \quad t \geq 0,
$$

where $q$ is a polynomial of degree $n-1$.

Proof. Since $y \in \Lambda_{n 1}$ we may parametrize $y$ in the form

$$
y(t)=Y(\mathbf{c}, \lambda, t)=\sum_{i=1}^{l} \sum_{j=1}^{k_{i}} c_{i j} t^{j-1} \exp \left(\lambda_{i} t\right)
$$


where $\lambda_{1}<\lambda_{2}<\ldots<\lambda_{l} \leq-1$ with $1 \leq l \leq n$, where $k_{1}, k_{2}, \cdots, k_{l}$ are positive integers with sum $n$, and where $c, \lambda$ denote the $n$ coefficients $c_{i j}$ and the $l$ exponential parameters $\lambda_{i}$, respectively.

Let $z_{1}<z_{2}<\ldots<z_{m}$ be the zeros of $y$ in $(0, \tau)$ where $y$ changes algebraic sign (if any such zeros exist). We shall show that $m=n-1$. Indeed if $m<n-1$, then we can find coefficients $\mathbf{c}^{*} \neq \mathbf{0}$ so that the function

$$
b(t)=Y\left(\mathrm{c}^{*}, \lambda, t\right), \quad t \geq 0,
$$

will have $n-1$ zeros with $m$ of these zeros located at the points $z_{1}, z_{2}$, $\cdots, z_{m}$ and the remaining $n-1-m$ located near $\tau$ in such a manner that

$$
\begin{aligned}
& \operatorname{sgn} h(t)=-\operatorname{sgn} y(t) \quad \text { whenever } \gamma_{n}(t) \geq\left[1+\gamma_{n}(\tau)\right] / 2, \\
& \operatorname{sgn} b(\tau)=\operatorname{sgn} y(\tau)
\end{aligned}
$$

(where

$$
\operatorname{sgn} x= \begin{cases}0 & \text { if } x=0 \\ x /|x| & \text { if } x \neq 0\end{cases}
$$

is the signum function). This will always be possible since the set of basis functions

$$
\phi_{i j}(t)=t^{j-1} \exp \left(\lambda_{i} t\right), \quad j=1,2, \cdots, k_{i} ; i=1,2, \cdots, l,
$$

appearing in (19) forms a Haar system of order $n$, cf. [3, p. 177]. But if (17), (20), (21) hold, then for all sufficiently small $\epsilon>0$ the function

$$
y_{\epsilon}(t)=Y\left(\mathbf{c}+\epsilon \mathbf{c}^{*}, \lambda, t\right)=y(t)+\epsilon b(t)
$$

will simultaneously have the properties

$$
\begin{gathered}
y_{\epsilon} \in \Lambda_{n 1} \quad \text { with }\left\|y_{\epsilon}\right\|<1, \\
y_{\epsilon}(\tau)>\gamma_{n}(\tau)
\end{gathered}
$$

in contradiction to (16). Thus we conclude $m=n-1$ and that $c_{i k_{i}} \neq 0$ for each $i=1,2, \ldots, l$ (since the system (22) which remains when $\phi_{i k_{i}}$ is removed is a Haar system of order $n-1)$.

Under these circumstances we can find parameters $\mathbf{c}^{*}$ and $\lambda^{*}=\left(\lambda_{1}^{*}\right.$, $0,0, \cdots, 0)$ such that if 


$$
b(t)=Y\left(\mathbf{c}^{*}, \lambda, t\right)+\lambda_{1}^{*} \sum_{j=1}^{k_{1}} c_{1 j} t^{j} \exp \left(\lambda_{1} t\right)
$$

then (21) holds. Again this is possible because (25) represents an arbitrary linear combination of the $n$ basis functions $\phi_{i j}$ of (22) and (since $c_{1 k} \neq$ 0 ) the corresponding function

$$
\phi_{1, k}+1
$$

which together form a Haar system of order $n+1$. This being the case we see that if $\lambda_{1}<-1$ then for all sufficiently small $\epsilon>0$ the function

$$
y_{\epsilon}(t)=Y\left(\mathbf{c}+\epsilon \mathbf{c}^{*}, \lambda+\epsilon \lambda^{*}, t\right)=y(t)+\epsilon b(t)+o(\epsilon)
$$

will again simultaneously have the properties (23) and (24) which is impossible. Hence $\lambda_{1}=-1$ and we conclude that $y$ has the form (18).

We shall now let

$$
q_{n}(t)=a_{n 0}+a_{n 1} t+\cdots+a_{n n} t^{n}, \quad n=0,1, \cdots,
$$

denote the Chebyshev polynomial of degree $n$ with respect to the semi-infinite interval $[0,+\infty)$ and the weight function $w(t)=\exp (-t)$, i.e., $q_{n}$ is selected so that the leading coefficient $a_{n n}$ in (26) is maximized subject to the constraint that $\max \left\{\left|q_{n}(t)\right| \exp (-t): t \geq 0\right\} \leq 1$, cf. [2]. Using slight variations of standard arguments (cf. [4, pp. 34-52]) it can be shown that this condition uniquely determines $q_{n}$ and that there is a unique set of extreme points $0=\tau_{n 0}<\tau_{n 1}<\cdots<\tau_{n n}$ such that

$$
q_{n}\left(\tau_{n i}\right) \exp \left(-\tau_{n i}\right)=(-1)^{n-i}, \quad i=0,1, \cdots, n
$$

Lemma 3. The envelope functions defined by (16) can be written in the form

$$
\gamma_{n+1}(t)=\left\{\begin{array}{ll}
1 & \text { if } 0 \leq t \leq \tau_{n n}, \\
q_{n}(t) \exp (-t) & \text { if } t \geq \tau_{n n},
\end{array} \quad n=0,1, \cdots .\right.
$$

Proof. Let $y_{n}(t)=q_{n}(t) \exp (-t), t \geq 0$, so that by construction $y_{n} \epsilon$ $\Lambda_{n+1,1}$ with $\left\|y_{n}\right\|=1$ and (using (27))

$$
y_{n}\left(\tau_{n i}\right)=(-1)^{n-i}, \quad i=0,1, \cdots, n .
$$

From (16) and (29) we see that $\gamma_{n+1}\left(\tau_{n n}\right)=1$, and since $\gamma_{n+1}$ is nonincreasing we obtain (28) for the case where $t \leq \tau_{n n}$. 
Suppose now that $\tau_{n, n+1}>\tau_{n n}$ is chosen so large that $\gamma_{n+1}\left(\tau_{n, n+1}\right)<$ 1. Using Lemma 2 we infer the existence of some polynomial $q$ of degree $n$ such that if $y(t)=q(t) \exp (-t)$ then $y \in \Lambda_{n+1,1}$ with $\|y\|=1$ and

$$
y\left(\tau_{n, n+1}\right)=\gamma_{n}\left(\tau_{n, n+1}\right) \geq y_{n}\left(\tau_{n, n+1}\right) .
$$

Using (29), (30) and the normalization conditions $\|y\|=\left\|y_{n}\right\|=1$ we find

$$
(-1)^{n-i}\left[y\left(\tau_{n i}\right)-y_{n}\left(\tau_{n i}\right)\right] \leq 0, \quad i=0,1, \cdots, n+1,
$$

and by removing the exponential factors we have

$$
(-1)^{n-i}\left[q\left(\tau_{n i}\right)-q_{n}\left(\tau_{n i}\right)\right] \leq 0, \quad i=0,1, \cdots, n+1,
$$

i.e. the polynomial $q-q_{n}$ of degree at most $n$ is alternately nonnegative and nonpositive on the $n+2$ distinct points $\tau_{n i}$. It follows that $q-q_{n} \equiv$ 0 and thus that (28) holds whenever $t>\tau_{n n}$ and $\gamma_{n+1}(t)<1$. But since $\gamma_{n+1}$ is continuous and since $y_{n}(t)<1$ for all $t>\tau_{n n}$, it follows that (28) holds for all $t \geq \tau_{n n}$.

The results of this section may be summarized as follows.

Theorem 2. Let $y \in \Lambda_{n a}$. Then

$$
|y(t)| \leq\|y\| \gamma_{n}(\alpha t), \quad t \geq 0
$$

where $\gamma_{n}$ is given by (28) and where

$$
\|y\|=\max \left\{|y(t)|: 0 \leq t \leq \tau_{n-1, n-1}\right\} .
$$

Moreover, the bound (31) is the best possible.

\section{REFERENCES}

1. D. W. Kammler, Existence of best approximations by sums of exponentials, J. Approximation Theory 9 (1973), 78-90.

2. - Chebyshev polynomials corresponding to a semi-infinite interval and an exponential weight factor, Math. Comp. 27 (1973), 633-637.

3. G. Meinardus, Approximation of functions: Theory and numerical methods, Springer Tracts in Natural Philosophy, vol. 13, Springer-Verlag, New York, 1967. MR 36 \# 571.

4. I. P. Natanson, Constructive function theory. Vol. I. Uniform approximation, GITTL, Moscow, 1949; English transl., Ungar, New York, 1964. MR 33 \#4529a.

DEP ARTMENT OF MATHEMATICS, SOUTHERN ILLINOIS UNIVERSITY, CARBONDALE, ILLINOIS 62901 